\title{
Remifentanil-based propofol-supplemented vs. balanced sevoflurane-sufentanil anesthesia regimens on bispectral index recovery after cardiac surgery: a randomized controlled study
}

\author{
Tae-Yun Sung ${ }^{1}$, Dong-Kyu Lee ${ }^{2}$, Jiyon Bang ${ }^{3}$, Jimin Choi ${ }^{4}$, \\ Saemi Shin ${ }^{5}$, and Tae-Yop Kim ${ }^{4}$
}

'Department of Anesthesiology and Pain Medicine, Konyang University Hospital, College of Medicine, Konyang University, Daejeon, ${ }^{2}$ Department of Anesthesiology, Korea University Guro Hospital, Korea University College of Medicine, ${ }^{3}$ Department of Anesthesiology and Pain Medicine, Asan Medical Center, University of Ulsan College of Medicine, ${ }^{4}$ Department of Anesthesiology, Konkuk University Medical

Received May 19, 2020

Revised July 1, 2020

Accepted July 16, 2020

\author{
Corresponding author \\ Tae-Yop Kim, M.D., Ph.D. \\ Department of Anesthesiology, \\ Konkuk University Medical Center, \\ 120-1 Neungdong-ro, Gwangjin-gu, \\ Seoul 05030, Korea \\ Tel: 82-2-2030-5445 \\ Fax: 82-2-2030-5449 \\ E-mail: taeyop@gmail.com
}

Background: The present study was to compare the potential impact of remifentanil-based propofol-supplemented anesthesia regimen vs. conventional sevoflurane-sufentanil balanced anesthesia on postoperative recovery of consciousness indicated by bispectral index (BIS) values in patients undergoing cardiac surgery.

Methods: Patients undergoing cardiac surgery were randomly allocated to get the remifentanil-based propofol-supplemented anesthesia employing target-controlled infusion ( $\mathrm{TCl}$ ) of remifentanil and propofol (Group-PR, $n=15$ ) or a balanced-anesthesia employing sevoflurane-inhalation and TCl-sufentanil (Group-C, $n=19$ ). In Group-PR, plasma concentration (Cp) of TCl-remifentanil was fixed at $20 \mathrm{ng} / \mathrm{ml}$, and the effect-site concentration of TCl-propofol was adjusted within $0.8-2.0 \mu \mathrm{g} / \mathrm{ml}$ to maintain BIS value of 40-60. In Group-C, sevoflurane dosage was adjusted within 1-1.5 minimum alveolar concentration to maintain BIS of 4060 , and $\mathrm{Cp}$ of TCl-sufentanil was fixed at $0.4 \mathrm{ng} / \mathrm{ml}$. The inter-group difference in the time for achieving postoperative BIS > 80 (T-BIS80) in the intensive care unit was determined as the primary outcome. The inter-group difference in the extubation time was determined as the secondary outcome.

Results: T-BIS80, was shorter in Group-PR than Group-C (121.4 \pm 64.9 min vs. $182.9 \pm$ $85.1 \mathrm{~min}$, respectively; the difference of means $-61.5 \mathrm{~min}$; $95 \% \mathrm{Cl}-115.7$ to $-7.4 \mathrm{~min}$; effect size 0.812; $P=0.027)$. The extubation time was shorter in Group-PR than in Group-C (434.7 $\pm 131.3 \mathrm{~min}$ vs. $946.6 \pm 393.3 \mathrm{~min}$, respectively, $\mathrm{P}<0.001)$.

Conclusions: Compared with the conventional sevoflurane-sufentanil balanced anesthesia, the remifentanil-based propofol-supplemented anesthesia showed significantly faster postoperative conscious recovery in patients undergoing cardiac surgery.

Keywords: Anesthetics; Consciousness; Propofol; Remifentanil.

This is an Open Access article distributed under the terms of the Creative Commons Attribution Non-Commercial License (http://creativecommons.org/licenses/by-nc/4.0) which permits unrestricted non-commercial use, distribution, and reproduction in any medium, provided the original work is properly cited.

Copyright (C) the Korean Society of Anesthesiologists, 2020 


\section{INTRODUCTION}

While various anesthesia regimens can be employed for cardiac anesthesia, the postoperative recovery is dependent on the time to achieve wear-off of anesthetic agents and adequate conscious recovery and spontaneous ventilation. The patient's conscious recovery speed is one of the major factors for determining overall postoperative recovery [1].

Propofol-remifentanil combination can be employed as total intravenous anesthesia (TIVA) for cardiac surgery, probably due to their shorter duration of clinical effects providing faster recovery [2,3]. Remifentanil is an ultra-short-acting with a very short context-sensitive half-life, and it has a supra-additive interaction with propofol regarding hypnotic and analgesic effects $[4,5]$. Administering remifentanil-based anesthesia employing a high intraoperative dosage of remifentanil, even during a prolonged period, would not compromise postoperative recovery in cardiac surgery. Likewise, administering propofol during a brief period usually does not show any dose-dependent difference in recovery speed. However, administering propofol during a prolonged period, especially in higher dosage, may compromise the patient's overall recovery, because propofol has a relatively long context-sensitive half-life and variable elimination half-life $[2,3,6]$. Bindra et al. [7] showed that sevoflurane could be preferred to propofol for achieving rapid emergence and cognitive recovery, even in non-cardiac surgery. Therefore, a strategy for reducing or minimizing propofol dosage would be beneficial for reducing the potential delay in postoperative recovery after applying the propofol-remifentanil combined regimen. Of course, the degree of the dosage reduction must be adjusted to avoid intraoperative awareness.

If a balanced regimen employing sevoflurane and sufentanil is used, sufentanil's longer duration of action would be a major determinant for the speed of postoperative recovery $[8,9]$.

We assumed that the remifentanil-based regimen supplemented by the reduced dosage of propofol would provide relatively faster conscious recovery compared with the balanced sevoflurane and sufentanil regimen in patients undergoing cardiac surgery.

Intraoperative electroencephalography (EEG)-based sedation monitors, including bispectral index (BIS), have been used for assessing the depth of sedation (hypnosis) and titrating the dosage of anesthetic (hypnotic) agents.
Despite controversies and its limitations [10-12], several investigations speculated the efficacy of BIS, as an objective modality for assessing the depth of sedation and managing patient sedation in the intensive care unit (ICU) $[4,5,10,12]$. Most of all, BIS does not require physical or auditory stimulus, which is necessarily used in assessing the depth of sedation by using other subjective modalities but interrupts the already established patients' sedation level. This feature enables objective and seamless evaluation of postoperative sedation in the ICU $[4,5]$.

Our study compared the time of achieving the BIS value $>80$ in the ICU after applying two different anesthesia regimens, a remifentanil-based propofol-supplemented regimen vs. a conventional sevoflurane-sufentanil balanced anesthesia for cardiac surgery. The extubation times of the two different anesthesia regimens were also compared, as a secondary outcome of our study.

\section{MATERIALS AND METHODS}

\section{Population and study protocol}

After obtaining the Institutional Review Board approval (no. KUH1160080), the informed consent was obtained from all patients before starting the study. This prospective, randomized, and controlled trial was registered to clinicaltrials.org (no. NCT02400879). Patients undergoing elective cardiac valve repair or replacement surgery were included in this study.

Thirty-eight patients were randomly allocated (allocation ratio 1:1) into two groups using sealed envelope method: remifentanil-based propofol-supplemented anesthesia regimen was applied in Group-PR ( $\mathrm{n}=19)$ and conventional sevoflurane-sufentanil balanced anesthesia regimen was applied in Group-C ( $\mathrm{n}=19)$. All patient was blinded to their allocation.

Preoperative and intraoperative exclusion criteria were applied. Preoperative exclusion criteria were 1) urgent or emergent surgery, 2) left ventricle ejection fraction $<50 \%$, 3) application of intra-aortic balloon pump (IABP), 4) myocardial infarction, 5) neurologic deficit or cognitive impairment, 6) chronic or acute pulmonary disease, 7) hepatic or renal impairment, 8) cardiac pacing, 9) inotropic medication.

Intraoperative exclusion criteria were 1) cardiopulmonary bypass (CPB) application of > $300 \mathrm{~min}, 2)$ transfusion of packed red blood cell of $>5$ units, 3) post-CPB use of 
double inotropic support for > $30 \mathrm{~min}, 4)$ post-CPB pacing, 5) intra- and post-operative IABP application, 6) postoperative hemodialysis, 7) number of administration of additional propofol $\geq 2,8$ ) postoperative use of sedatives (e.g., midazolam, propofol) before extubation, 9) excessive bleeding of $>500 \mathrm{ml}$ during postoperative $6 \mathrm{~h}, 10$ ) reoperation due to excessive bleeding.

\section{Anesthesia regimens}

After establishing a routine invasive arterial blood pressure and a noninvasive patient monitoring such as pulse oximetry $\left(\mathrm{SpO}_{2}\right)$, electrocardiography, BIS (BIS XP monitor, Model A2000, Aspect Medical Systems, USA) and cerebral oximetry, anesthesia was induced by two groups of anesthesiologists each consisting of two anesthesiologists (AA and $\mathrm{BB}$ ) using different regimens: a target-controlled infusion (TCI) of propofol (effect-site concentration [Ce] of 1.0$2.0 \mu \mathrm{g} / \mathrm{ml}$ ) and remifentanil (plasma concentration [Cp], $20 \mathrm{ng} / \mathrm{ml}$ with the time to reach target concentration of 7 min) in Group-PR (by AA); and anesthesia was induced by a bolus injection of thiopental sodium $3-4 \mathrm{mg} / \mathrm{kg}$ and TCI-sufentanil (Cp of $0.4 \mathrm{ng} / \mathrm{ml}$ ) in Group-C (by BB). Tracheal intubation was facilitated by bolus rocuronium under the guidance of neuromuscular train-of-four monitoring in both groups. For anesthesia maintenance, TCI-remifentanil (fixed $\mathrm{Cp}$ of $20 \mathrm{ng} / \mathrm{ml}$ ) and TCI-propofol (variable Ce $0.8-2.0 \mu \mathrm{g} / \mathrm{ml}$ to maintain BIS of 40-60) was used in Group-PR, and TCI-sufentanil (fixed Cp of $0.4 \mathrm{ng} /$ $\mathrm{ml}$ ) and sevoflurane (variable 1.0-1.5 minimum alveolar concentration [11] to maintain BIS of 40-60) was used in Group-C.

Hemodynamic parameters including mean blood pressure (MBP) and heart rate were maintained within 80$120 \%$ of preoperative value in both groups. Neuromuscular block during the operation was achieved by continuous infusion of rocuronium ( $3 \mu \mathrm{g} / \mathrm{kg} / \mathrm{min}$ ) in both groups.

Controlled ventilation of $\mathrm{O}_{2}$ /air mixture $\left(\mathrm{FiO}_{2}\right.$ 0.5-0.6) was applied with the following settings: tidal volume of 6-7 $\mathrm{ml} /$ ideal body weight, respiratory rate maintaining the state of normocapnia (end-tidal $\mathrm{CO}_{2}$ tension 35-40 $\mathrm{mmHg}$ ), and inspiration to expiration ratio of $1: 2$. The intermittent lung-recruit maneuver was applied with constant application of positive end-expiratory pressure of 6-8 mmHg.

Pulmonary artery catheter and a probe of transesophageal echocardiography were placed after the anesthesia in- duction. All surgeries were performed by one surgeon and four surgical assistants. They were blinded to patient allocation and the purpose of this study. After completing a sternotomy and administering 300 units/kg of heparin, arterial and venous cannulations for CPB were performed at activated clotting time $>400-450$ s and CPB was conducted using a reservoir, a membrane oxygenator, a roller pump, and a heat exchanger. CPB flow, MBP, and hemodilution were adjusted to maintain the values of cerebral oximetry within $120 \%$ of preinduction values during the CPB period.

During surgery, if the BIS value exceeded 60 for 3 min and persisted against the increased dosage of sevoflurane to 1.5 MAC in Group-C or Ce of propofol to $2.0 \mu \mathrm{g} / \mathrm{ml}$ in Group-PR, bolus propofol 10-20 mg was administered.

\section{Protocols of ventilator weaning and extubation in the ICU}

After the surgery, patients were transferred to the ICU in intubated status and received controlled or assisted ventilation till the time of extubation. For the postoperative intravenous patient-control analgesia (IV-PCA) in Group-PR, remifentanil infusion of $0.25-0.3 \mu \mathrm{g} / \mathrm{kg} / \mathrm{min}$ was continued till the time of performing extubation. According to the institutional protocols, the IV-PCA of Group-PR was started at the decision of performing extubation; in contrast, it in Group-C was started at the time of the sternum closure. For the IV-PCA in Group-PR, the alfentanil-ketorolac-ondansetron combination was set to deliver alfentanil of $1.0 \mu \mathrm{g} /$ $\mathrm{kg} / \mathrm{h}$ and a bolus dose of alfentanil $1.0 \mu \mathrm{g} / \mathrm{kg}$ with a $10-\mathrm{min}$ lockout interval. For the IV-PCA in Group-C, the fentanyl-ketorolac-ondansetron combination was set to fentanyl of $0.2 \mu \mathrm{g} / \mathrm{kg} / \mathrm{h}$ and a bolus dose fentanyl $0.2 \mu \mathrm{g} / \mathrm{kg}$ with 15 min lockout interval.

The mode of the ventilator was converted from volume-controlled ventilation to synchronized intermittent mandatory ventilation (SIMV) when the following criteria were met: stable hemodynamics, spontaneous respiration, and the respiratory rate $10-25$ breaths $/ \mathrm{min}$.

The decision for tracheal extubation was made when the following criteria were met: awake state (or BIS > 80), stable hemodynamics, normal airway reflex, respiratory rate of 10-25 breaths/min, $\mathrm{SpO}_{2}>95 \%$ at $\mathrm{FiO}_{2}<0.6, \mathrm{pH} \geq 7.3$ and $\mathrm{PaCO}_{2}<55 \mathrm{mmHg}$. Extubation was performed at 20 min after the decision in all patients. In Group-PR, the IVPCA was started at the decision for extubation, and the 
remifentanil infusion was stopped immediately before the extubation.

\section{Measurements}

Operation time, CPB time, aortic cross-clamp (ACC) time, intraoperative fluid administration quantity, transfusion requirements, intraoperative urine output, hematocrit at admission to the ICU, preoperative and postoperative $\mathrm{PaO}_{2} / \mathrm{FiO}_{2}$ ratio were recorded. In the ICU, patients who were not calm despite the frequent verbal instructions and who were restless or combative, excited, disoriented, and required physical restraints were regarded as "agitated." The incidences of agitation were also recorded.

As a primary outcome of this study, the duration from the end of the surgery, which is defined as the completion of skin closure, to the time of achieving the BIS value $>80$ (persisting $>3 \mathrm{~min}, \mathrm{~T}$-BIS80) was measured in the ICU $[5,13]$.

The duration from the end of surgery to the time of extubation (T-extubation) and the duration from the end of surgery to the time of initiating SIMV (T-SIMV) was also measured in the ICU.

All data were collected by trained observers who did not participate in patient care and were blinded to the current study.

\section{Statistical analysis}

The primary outcome variable was T-BIS80. According to our pilot test results $(\mathrm{n}=10)$, mean and standard deviation were 117.2 and $47.6 \mathrm{~min}$ for Group-C, and 69.6 and $40 \mathrm{~min}$ for Group-PR. Based on this pilot test result, the estimated effect size was 1.083, assuming a error probability; 0.05 (2-tailed), power; 0.80 , allocation ratio; $1: 1$, the calculated sample size was 30 .

The required sample size was 38 (19 for each group) to fulfill the study protocol assumption, considering the $20 \%$ drop out rate.

Statistical analysis was performed using Sigmastat version 3.1 (Systat Software Inc., USA). After performing the normality test using the Shapiro-Wilk test, continuous variables were analyzed using a $t$-test (2-tailed) or the Mann-Whitney $U$ test. Categorical variables were analyzed by a chi-square test or a Fisher's exact test, as applicable. The data collected were expressed as mean and standard deviation, median (1Q, 3Q), or numbers of patients (\%). A
$\mathrm{P}$ value $<0.05$ was considered significant.

\section{RESULTS}

A total of 43 patients was evaluated for eligibility. According to the inclusion and preoperative exclusion criteria, five patients were excluded (3 declined to participate, 2 had hepatic or renal impairment), and 38 patients were included in this study. Additionally, four patients from Group-PR were withdrawn because one patient required post-CPB cardiac pacing, and three patients required postCPB use of double inotropic support > $30 \mathrm{~min}$. Thus, 34 patients (19 for Group-C and 15 for Group-PR) were included in the statistical analysis (Fig. 1).

The patients' demographic profiles and surgical types did not show intergroup differences (Table 1). Preoperative hematocrit, $\mathrm{PaO}_{2} / \mathrm{FiO}_{2}$, $\mathrm{pH}$, electrolyte, lactate, and glucose were comparable between the two groups (Table 2). Duration of surgery in Group-PR was significantly longer than that in Group-C $(445 \pm 151$ min vs. $319 \pm 64$ min, respectively, the difference of means $126 \mathrm{~min}$; $95 \%$ confidence interval [95\% CI] 39-214 min; effect size 1.094; $\mathrm{P}=0.007$ ) (Table 3). CPB and ACC time, intraoperative fluid administration quantity, transfusion requirements, intraoperative urine output, and the number of patients who received additional propofol did not show intergroup differences (Table 3).

At the time of admission to the ICU, BIS, hematocrit, $\mathrm{PaO}_{2} / \mathrm{FiO}_{2}, \mathrm{pH}$, electrolyte, and lactate did not show intergroup differences. However, at the time of admission to the ICU, glucose level was significantly lower in Group-PR than in group C (109.7 \pm 53.0 vs. $163.9 \pm 51.7 \mathrm{mg} / \mathrm{dl}$, respectively; differences of means $-54.2 \mathrm{mg} / \mathrm{dl} ; 95 \% \mathrm{CI}-91.0$ to -17.5 $\mathrm{mg} / \mathrm{dl}$; effect size 1.035; $\mathrm{P}=0.005)$.

The primary outcome variable, T-BIS80, was significantly shorter in Group-PR compared to Group-C (121.4 \pm 64.9 min vs. $182.9 \pm 85.1 \mathrm{~min}$, respectively; the difference of means $-61.5 \mathrm{~min}$; $95 \% \mathrm{CI}-115.7$ to $-7.4 \mathrm{~min}$; effect size 0.812; $\mathrm{P}=0.027$ ).

As the secondary outcome variable, T-extubation in Group-PR was significantly shorter than that in Group-C $(434.7 \pm 131.3 \mathrm{~min}$ vs. $946.6 \pm 393.3 \mathrm{~min}$, respectively; differences of means $-511.9 \mathrm{~min}$; $95 \%$ CI -711.4 to $-312.4 \mathrm{~min}$; effect size 1.746; $\mathrm{P}<0.001)$. T-SIMV did not show inter-group differences.

The incidence of agitation in the ICU did not show intergroup differences (Table 4). 


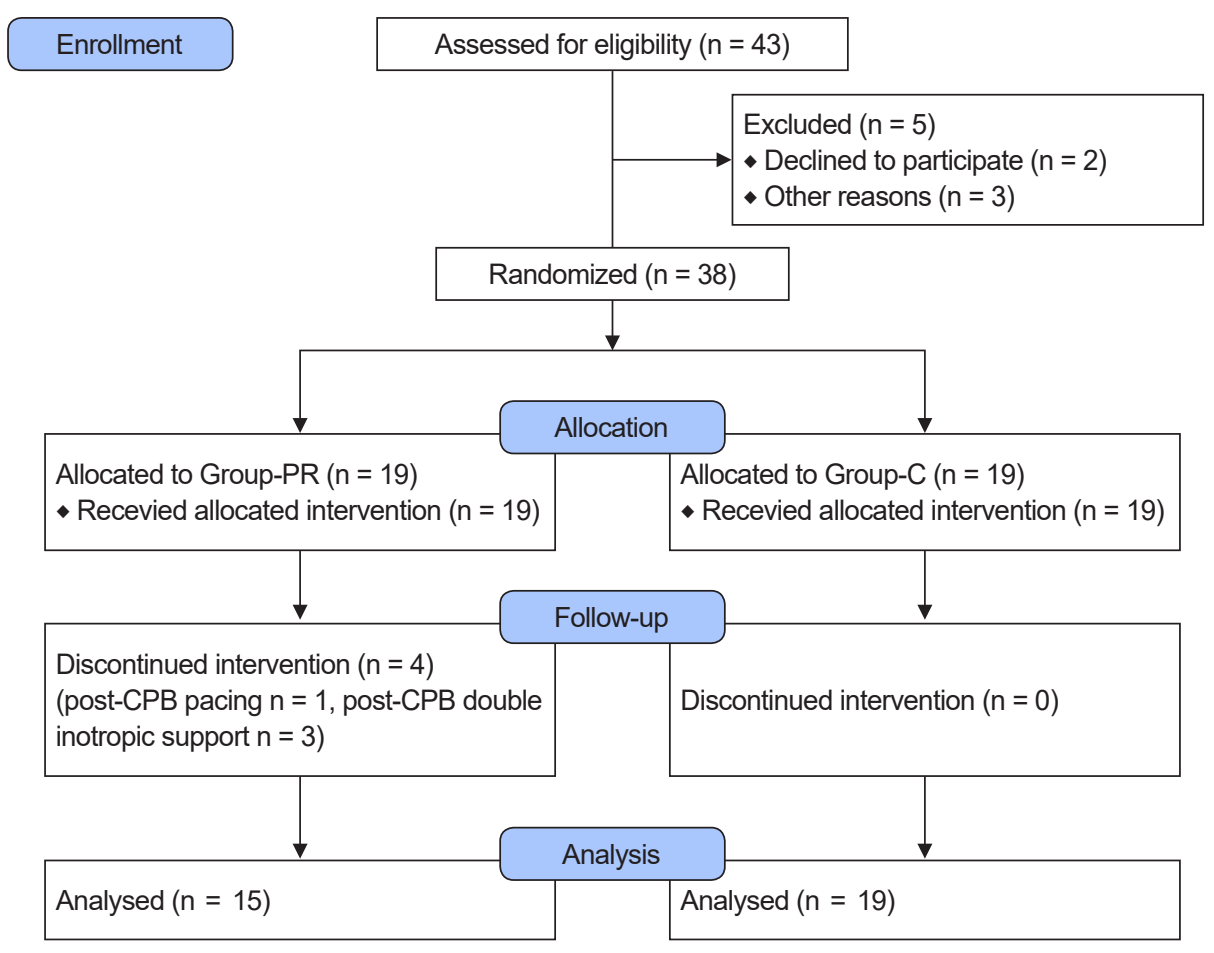

Fig. 1. Flow chart. Thirty-eight patients were randomly allocated into the Group-PR or Group-C, and four patients in the Group-PR were excluded because one patient required post-CPB cardiac pacing, and 3 patients were applied post-CPB use of double inotropic support. Group-PR: remifentanil-based propofol-supplemented regimen, Group-C: combined sevoflurane-sufentanil regimen, CPB: cardiopulmonary bypass.

Table 1. Demographic Data

\begin{tabular}{|c|c|c|c|}
\hline Variable & Group-PR $(n=15)$ & Group-C $(n=19)$ & $P$ value \\
\hline Age (yr) & $49.5 \pm 14.8$ & $47.0 \pm 13.7$ & 0.618 \\
\hline $\operatorname{Sex}(M / F)$ & $8 / 7$ & $10 / 9$ & 0.986 \\
\hline Height (cm) & $162.6 \pm 7.8$ & $166.4 \pm 8.8$ & 0.201 \\
\hline Weight (kg) & $55.0(50.0,65.0)$ & $63.8(58.0,68.0)$ & 0.058 \\
\hline \multicolumn{4}{|l|}{ Present illness } \\
\hline Diabetes mellitus & $0(0)$ & $1(5.3)$ & 1.000 \\
\hline Hypertension & $7(46.7)$ & $6(31.6)$ & 0.070 \\
\hline Diabetes and hypertension & $0(0)$ & $0(0)$ & NA \\
\hline \multicolumn{4}{|l|}{ Previous medication } \\
\hline Angiotensin blocker & $5(33.3)$ & $4(21.1)$ & 0.462 \\
\hline ACE-inhibitor & $4(26.7)$ & $1(5.3)$ & 0.146 \\
\hline Calcium channel blocker & $3(20.0)$ & $0(0)$ & 0.076 \\
\hline Beta-blocker & $3(20.0)$ & $3(15.8)$ & 1.000 \\
\hline Statin & $2(13.3)$ & $1(5.3)$ & 0.571 \\
\hline Warfarin & $2(13.3)$ & $3(15.8)$ & 1.000 \\
\hline Operation type & & & 0.934 \\
\hline Mitral valve repair & $6(40.0)$ & $9(47.4)$ & \\
\hline Aortic valve repair & $5(33.3)$ & $4(21.1)$ & \\
\hline Double valve repair & $4(26.7)$ & $6(31.6)$ & \\
\hline
\end{tabular}

Values are presented as mean \pm SD, numbers of patients (\%), or median (1Q, 3Q). Group-PR: propofol-remifentanil regimen, Group-C: sevoflurane-sufentanil regimen, ACE: angiotensin-converting enzyme, NA: not applicable. 
Table 2. Preoperative Variables

\begin{tabular}{lccc}
\hline \multicolumn{1}{c}{ Variable } & Group-PR $(\mathrm{n}=15)$ & Group-C $(\mathrm{n}=19)$ & $\mathrm{P}$ value \\
\hline Hematocrit $(\%)$ & $38.0(35.0,40.0)$ & $35.5(33.0,38.0)$ & 0.081 \\
$\mathrm{PaO}_{2} / \mathrm{FiO}_{2}$ ratio & $362 \pm 164$ & $451 \pm 208$ & 0.182 \\
$\mathrm{pH}$ & $7.43(7.40,7.46)$ & $7.44(7.41,7.45)$ & 0.476 \\
$\mathrm{Na}^{+}(\mathrm{mmol} / \mathrm{L})$ & $140(138,140)$ & $140(138,142)$ & 0.716 \\
$\mathrm{~K}^{+}(\mathrm{mmol} / \mathrm{L})$ & $3.8(3.6,4.1)$ & $3.7(3.5,4.0)$ & 0.544 \\
$\mathrm{Ca}^{++}(\mathrm{mg} / \mathrm{dl})$ & $1.1(1.1,1.1)$ & $1.1(1.1,1.2)$ & 0.082 \\
$\mathrm{Mg}^{++}(\mathrm{mmol} / \mathrm{L})$ & $0.5(0.5,0.6)$ & $0.5(0.5,0.7)$ & 0.689 \\
Lactate $(\mathrm{mmol} / \mathrm{L})$ & $0.7(0.7,1.5)$ & $0.8(0.7,1.1)$ & 0.622 \\
Glucose $(\mathrm{mg} / \mathrm{dl})$ & $101(94,119)$ & $119(100,137)$ & 0.138 \\
\hline
\end{tabular}

Values are presented as median (1Q, 3Q) or mean \pm SD. Values are measured just after anesthesia induction. Group-PR: propofol-remifentanil regimen, Group-C: sevoflurane-sufentanil regimen, $\mathrm{PaO}_{2} / \mathrm{FiO}_{2}$ ratio: partial pressure of $\mathrm{O}_{2}$ in arterial blood/the fraction (percent) of inspired $\mathrm{O}_{2}$ ratio.

Table 3. Intraoperative Variables

\begin{tabular}{|c|c|c|c|c|}
\hline Variable & Group-PR $(n=15)$ & Group-C $(n=19)$ & Difference of means $(95 \% \mathrm{Cl})$ & $P$ value \\
\hline Duration of surgery (min) & $445 \pm 151$ & $319 \pm 64$ & $126(39-214)$ & 0.007 \\
\hline CPB time & $155 \pm 60$ & $143 \pm 56$ & NA & 0.535 \\
\hline ACC time & $94 \pm 50$ & $82 \pm 41$ & NA & 0.450 \\
\hline Intraoperative fluid (ml) & $1,460 \pm 409$ & $1,537 \pm 682$ & NA & 0.703 \\
\hline Crystalloid & $907 \pm 187$ & $942 \pm 413$ & NA & 0.760 \\
\hline Colloid & $553 \pm 302$ & $595 \pm 387$ & NA & 0.736 \\
\hline Packed RBC (unit) & $2.3 \pm 2.2$ & $1.9 \pm 2.3$ & NA & 0.621 \\
\hline Intraoperative urine $(\mathrm{ml})$ & $1,257 \pm 496$ & $1,441 \pm 671$ & NA & 0.382 \\
\hline Additional propofol* & $2(13.3)$ & $1(5.3)$ & NA & 0.621 \\
\hline
\end{tabular}

Values are presented as mean \pm SD or numbers of patients (\%). Group-PR: propofol-remifentanil regimen, Group-C: sevoflurane-sufentanil regimen, 95\% Cl: 95\% confidence interval, CPB: cardiopulmonary bypass, ACC: aortic cross-clamp, NA: not applicable, BIS: bispectral index. *Number of patients required additional propofol bolus due to BIS value $>60$.

Table 4. Postoperative Variables in the Intensive Care Unit

\begin{tabular}{|c|c|c|c|c|}
\hline Variable & Group-PR ( $\mathrm{n}=15)$ & Group-C $(n=19)$ & Difference of means $(95 \% \mathrm{Cl})$ & $P$ value \\
\hline \multicolumn{5}{|l|}{ At admission* } \\
\hline $\mathrm{BIS}(0-100)$ & $47 \pm 5$ & $44 \pm 7$ & NA & 0.176 \\
\hline Hematocrit (\%) & $29(28,30)$ & $30(26,31)$ & NA & 0.662 \\
\hline $\mathrm{PaO}_{2} / \mathrm{FiO}_{2}$ ratio & $347(181,447)$ & $352(223,431)$ & NA & 0.986 \\
\hline $\mathrm{pH}$ & $7.42(7.39,7.45)$ & $7.43(7.39,7.44)$ & NA & 0.871 \\
\hline $\mathrm{Na}^{+}(\mathrm{mmol} / \mathrm{L})$ & $145(143,148)$ & $144(141,146)$ & NA & 0.296 \\
\hline $\mathrm{K}^{+}(\mathrm{mmol} / \mathrm{L})$ & $4.1(3.7,4.5)$ & $4.1(4.0,4.4)$ & NA & 0.802 \\
\hline $\mathrm{Ca}^{++}(\mathrm{mg} / \mathrm{dl})$ & $1.1 \pm 0.1$ & $1.1 \pm 0.1$ & NA & 0.413 \\
\hline $\mathrm{Mg}^{++}(\mathrm{mmol} / \mathrm{L})$ & $0.6 \pm 0.1$ & $0.7 \pm 0.1$ & NA & 0.129 \\
\hline Glucose (mg/dl) & $109.7 \pm 53.0$ & $163.9 \pm 51.7$ & $-54.2(-91.0$ to -17.5$)$ & 0.005 \\
\hline Lactate (mmol/L) & $2.2 \pm 1.0$ & $2.8 \pm 1.1$ & NA & 0.154 \\
\hline Time to BIS > 80 (min) & $121.4 \pm 64.9$ & $182.9 \pm 85.1$ & $-61.5(-115.7$ to -7.4$)$ & 0.027 \\
\hline Time to SIMV (min) & $352.8 \pm 136.2$ & $426.7 \pm 126.7$ & NA & 0.112 \\
\hline Time to extubation (min) & $434.7 \pm 131.3$ & $946.6 \pm 393.3$ & $-511.9(-711.4$ to -312.4$)$ & $<0.001$ \\
\hline Agitation & $7(46.7)$ & $6(31.6)$ & NA & 0.369 \\
\hline
\end{tabular}

Values are presented as mean $\pm \mathrm{SD}$, median (1Q, 3Q), or numbers of patients (\%). Group-PR: propofol-remifentanil regimen, Group-C: sevoflurane-sufentanil regimen, $95 \% \mathrm{Cl}$ : $95 \%$ confidence interval, BIS: bispectral index, SIMV: synchronized intermittent mandatory ventilation, $\mathrm{NA}$ : not applicable. * Just after admission to the intensive care unit, $\mathrm{PaO}_{2} / \mathrm{FiO}_{2}$ ratio: partial pressure of $\mathrm{O}_{2}$ in arterial blood/the fraction (percent) of inspired oxygen ratio. 


\section{DISCUSSION}

Our result demonstrated that the remifentanil-based propofol-supplemented regimen is superior to the conventional balanced-anesthesia regimen in providing a faster time to achieve postoperative BIS > 80 in cardiac surgery. Despite the random allocation and surgery by the same surgeon, the duration of surgery was longer in Group-PR. Considering that the longer duration of surgery is a risk factor for delayed patient awakening after anesthesia [14], our result with the shorter recovery despite the longer duration of surgery in Group-PR (445 min vs. $319 \mathrm{~min}$ in Group-C) may be of much more significant meaning.

So far, few studies have compared postoperative conscious recovery time after applying the remifentanil-based propofol-supplemented anesthesia regimens vs. conventional sevoflurane-sufentanil balanced anesthesia regimens for cardiac surgery. According to our result, remifentanil-based propofol-supplemented TIVA can be favored, especially for patients requiring fast postoperative conscious recovery in cardiac surgery.

We employed the BIS values $>80$, as the indicator of the patient's conscious recovery in the ICU, since previous reports showed that BIS values ranging $64-80$ corresponded to a transitional status from unconsciousness to consciousness [15]. BIS $>80$ was comparable to the sedation status responding to the command, "open your eyes" [13]. As mentioned earlier, BIS monitoring enables a seamless and objective evaluation of the depth of sedation in the ICU $[4,5]$. Our result indicating the remifentanil's superiority in providing faster BIS recovery corresponds to a previous study employing a subjective sedation scale, in which remifentanil-anesthesia provided a shallower depth of postoperative sedation than sufentanil-anesthesia did in cardiac surgery [8].

In the meantime, remifentanil or sufentanil alone cannot provide sufficient sedation, but they can potentiate the sedative effect of concomitantly administered propofol or sevoflurane, as the opioid-sedative synergism [16]. In our results, remifentanil and sufentanil could potentiate the effects and reduce the requirement concomitantly administered propofol or sevoflurane (Ce of propofol or MAC of sevoflurane) in the context of propofol-remifentanil synergy for producing intraoperative hypnosis [16]. The dosage of propofol was also guided by the BIS monitor to avoid intraoperative awareness. The reduced dosage of propofol in Group-PR, Ce of $<2.0 \mu \mathrm{g} / \mathrm{ml}$, might also be beneficial in reducing or minimizing the propofol-induced myocardial depressive effect in the propofol-remifentanil combination for cardiac surgery $[17,18]$.

In Group-PR, the increased dosage of remifentanil throughout anesthesia did not seem to produce any residual effect due to its very short plasma elimination half-life (8-10 $\mathrm{min}$ ) and a context-sensitive half-time (3-5 $\mathrm{min}$ ) [16] The reduced dosage of propofol $(\mathrm{Ce} 0.8-2.0 \mu \mathrm{g} / \mathrm{ml}$ ), which was similar to that of making patient awake (Ce $1.8 \pm 0.7$ $\mu \mathrm{g} / \mathrm{ml}$ ) in a previous study [19], also facilitated postoperative recovery. Only stopping of its infusion might provide immediate resolution of its effects.

Since sevoflurane has a short context-sensitive half time ( $<5 \mathrm{~min}$ ) and the time for conscious recovery $(<8 \mathrm{~min})$ [20], the dosage of sufentanil in Group-C and its duration of administration might be a critical factor for producing the inter-group difference in T-BIS80. Previous reports also showed residual effects after administering opioids, including sufentanil $[8,9]$.

However, the dosage of sufentanil in Group-C was still minimal, $\mathrm{Cp} 0.4 \mathrm{ng} / \mathrm{ml}$, and far less than the dosage producing postoperative residual effects in previous studies $[9,21]$. Therefore, the dosage and the duration of administration might play an essential role in terms of achieving faster postoperative recovery. Previous studies also supported this discrepancy upon longer vs. shorter period (e.g., in non-cardiac surgeries) of sufentanil infusion: the recovery profiles were not different between remifentanil and sufentanil anesthesia for thyroidectomy [3], and the extubation time was not different between remifentanil and sufentanil anesthesia for craniotomy [2]. Measures for reducing sufentanil's residual effect, such as earlier discontinuation of its infusion 30 min before the ending of the surgery, seem to be useful [2].

Most previous studies had focused on the contribution of respiratory recovery as an indicator of the overall postoperative recovery in evaluating anesthesia regimens [22]. The extubation time (less than $6-10 \mathrm{~h}$ ) has been used as a typical indicator for evaluating postoperative recovery and fast-track anesthesia protocol [22]. The earlier recovery of consciousness may contribute to the earlier extubation in our study.

The shorter extubation time in the present study supports the superiority of remifentanil-based regimen to other anesthesia regimens employing sufentanil or fentanyl in providing faster postoperative respiratory recovery in cardiac surgery. The extubation time in Group-PR was signifi- 
cantly shorter (7 h vs. 15 h in Group-C). Bhavsar et al. [23] showed that remifentanil regimen was more effective in reducing time to tracheal extubation and length of stay in the recovery area. Furthermore, the dosage of the intermediate-acting opioid (e.g., morphine, fentanyl, sufentanil) administered during the intraoperative period is also important: higher intraoperative dosage would prolong postoperative extubation time (duration of intubation, odds ratio, 1.54 per sufentanil $1 \mu \mathrm{g} / \mathrm{kg}$ increment) [22].

In our study, despite the significant inter-group difference in the extubation time, the time to switch to SIMV, another determinant for extubation time, was not significantly different. Lison et al. [8] showed no difference in the ventilatory weaning times after using remifentanil vs. sufentanil anesthesia cardiac surgery, although the extubation time was faster in remifentanil anesthesia. Bhavsar et al. [23] also showed that remifentanil anesthesia was not superior to a standard moderate- to high-dose sufentanil regimen in the duration of ventilatory support or ICU stay $[17,21,23]$. Furthermore, some reports showed that early extubation ( $<6$ postoperative hours) paradoxically increases ICU length of stay in cardiac patients [24,25].

This study had limitations. First, the inter-group difference in the regimens for postoperative pain control (remifentanil and alfentanil vs. fentanyl) and the start timings of the PCA might affect the degree of pain intensity as well as recovery characteristics. However, even the greater dosage of remifentanil in Group-PR (approximately 8-9 times greater than fentanyl in the equivalent-dosage calculation) provided a significantly earlier recovery. Second, we did not include the postoperative evaluation of the Ramsay score or the Riker sedation-agitation scale. These subjective sedation scales might help overcome the controversy of postoperative BIS monitoring in the ICU setting $[4,5]$. However, their application had the potential to annoy the sedated patients and abruptly change the stabilized BIS values. Third, since the BIS monitor only manifests cortical activity, which is mainly affected by the hypnotic agents [26], the possible sedative effect by the extra-cortical pathway (e.g., sedative effects by opioids) was not evaluated [27]. There might be an inter-group discrepancy in the BIS values indicating the conscious recovery and those corresponding to the same level of sedation/awakeness. The BIS value indicating the return of consciousness in using a propofol-remifentanil combination would be higher than that in using propofol alone, as in this study. In comparing the inter-group differences in postoperative BIS values, we have to consider the variation of residual effects (or offset speed) all each anesthetic agent and BIS monitor's capability to manifest cortical EEG activity. Finally, the possible impact of other variables on patient's recovery and the effects of each anesthesia method on clinical outcomes, such as mortality rate, length of stay in the ICU, or length of hospital stay, were not evaluated due to the limited sample size. It may be necessary to evaluate this through further studies employing a much larger sample size.

In conclusion, the remifentanil-based propofol-supplemented regimen, which employing the relatively reduced dosage of propofol and the increased dosage of remifentanil, provided significantly faster recovery of postoperative BIS value, than balanced sevoflurane-sufentanil anesthesia regimen did in patients undergoing cardiac surgeries. It also provided significantly faster extubation time. Further investigation may be warranted to determine whether the propofol- remifentanil-ratio affects the recovery speed of postoperative consciousness in using the propofol-remifentanil anesthesia regimen for cardiac surgery.

\section{CONFLICTS OF INTEREST}

No potential conflict of interest relevant to this article was reported.

\section{AUTHOR CONTRIBUTIONS}

Conceptualization: Tae-Yop Kim, Tae-Yun Sung. Data curation: Tae-Yun Sung. Formal analysis: Dong-Kyu Lee. Funding acquisition: Tae-Yop Kim. Investigation: Tae-Yop Kim, Tae-Yun Sung. Methodology: Tae-Yop Kim, Tae-Yun Sung, Dong-Kyu Lee, Jiyon Bang. Project administration and resource: Tae-Yop Kim, Tae-Yun Sung, Dong-Kyu Lee, Jiyon Bang, Jimin Choi, Saemi Shin. Software: Dong-Kyu Lee. Supervision: Tae-Yop Kim. Validation: all authors. Visualization: Tae-Yun Sung, Dong-Kyu Lee. Writing-original draft: Tae-Yop Kim, Tae-Yun Sung, Dong-Kyu Lee. Writing-review \& editing: Tae-Yop Kim, Tae-Yun Sung, DongKyu Lee, Jiyon Bang, Jimin Choi, Saemi Shin. Approval of final manuscript: all authors.

\section{ORCID}

Tae-Yun Sung, https://orcid.org/0000-0002-0714-1477

Dong-Kyu Lee, https://orcid.org/0000-0002-4068-2363

Jiyon Bang, https://orcid.org/0000-0002-1031-9539 
Jimin Choi, https://orcid.org/0000-0001-6787-3058

Saemi Shin, https://orcid.org/0000-0003-4154-8542

Tae-Yop Kim, https://orcid.org/0000-0003-0806-8969

\section{REFERENCES}

1. Delphin E, Jackson D, Gubenko Y, Botea A, Esrig B, Fritz W, et al. Sevoflurane provides earlier tracheal extubation and assessment of cognitive recovery than isoflurane in patients undergoing off-pump coronary artery bypass surgery. J Cardiothorac Vasc Anesth 2007; 21: 690-5.

2. Necib S, Tubach F, Peuch C, LeBihan E, Samain E, Mantz J, et al. PROMIFLUNIL trial group. Recovery from anesthesia after craniotomy for supratentorial tumors: comparison of propofol-remifentanil and sevoflurane-sufentanil (the PROMIFLUNIL trial). J Neurosurg Anesthesiol 2014; 26: 37-44.

3. Lentschener C, Ghimouz A, Bonnichon P, Pépion C, Gomola A, Ozier Y. Remifentanil-propofol vs. sufentanil-propofol: optimal combinations in clinical anesthesia. Acta Anaesthesiol Scand 2003; 47: 84-9.

4. Kern SE, Xie G, White JL, Egan TD. A response surface analysis of propofol-remifentanil pharmacodynamic interaction in volunteers. Anesthesiology 2004; 100: 1373-81.

5. Riker RR, Fraser GL, Simmons LE, Wilkins ML. Validating the sedation-agitation scale with the bispectral index and visual analog scale in adult ICU patients after cardiac surgery. Intensive Care Med 2001; 27: 853-8.

6. Sahinovic MM, Struys MMRF, Absalom AR. Clinical pharmacokinetics and pharmacodynamics of propofol. Clin Pharmacokinet 2018; 57: 1539-58.

7. Bindra TK, Bariar H, Kumar P, Gupta N. To compare propofol and sevoflurane for maintenance of anaesthesia on recovery characteristics and cognitive functions: a randomised control trial. J Clin Diagn Res 2019; 13: UC10-4.

8. Lison S, Schill M, Conzen P. Fast-track cardiac anesthesia: efficacy and safety of remifentanil versus sufentanil. J Cardiothorac Vasc Anesth 2007; 21: 35-40.

9. Vuyk J, Mertens MJ, Olofsen E, Burm AG, Bovill JG. Propofol anesthesia and rational opioid selection: determination of optimal EC50-EC95 propofol-opioid concentrations that assure adequate anesthesia and a rapid return of consciousness. Anesthesiology 1997; 87: 1549-62.

10. Finger RG, Mallmann C, Nedel WL. BIS monitoring in sedated, mechanically ventilated patients: right tool in the wrong patients? A meta-analysis. Intensive Care Med 2016; 42: 1086-7.

11. Shetty RM, Bellini A, Wijayatilake DS, Hamilton MA, Jain R, Karanth S, et al. BIS monitoring versus clinical assessment for sedation in mechanically ventilated adults in the intensive care unit and its impact on clinical outcomes and resource utilization. Cochrane Database Syst Rev 2018; 2: CD011240.

12. Bilgili B, Montoya JC, Layon AJ, Berger AL, Kirchner HL, Gupta LK, et al. Utilizing bi-spectral index (BIS) for the monitoring of sedated adult ICU patients: a systematic review. Minerva Anestesiol 2017; 83: 288-301.

13. Doi M, Gajraj RJ, Mantzaridis H, Kenny GN. Relationship between calculated blood concentration of propofol and electrophysiological variables during emergence from anaesthesia: comparison of bispectral index, spectral edge frequency, median frequency and auditory evoked potential index. Br J Anaesth 1997; 78: 180-4.

14. Misal US, Joshi SA, Shaikh MM. Delayed recovery from anesthesia: a postgraduate educational review. Anesth Essays Res 2016; 10: 164-72.

15. Wang ZH, Chen H, Yang YL, Shi ZH, Guo QH, Li YW, et al. Bispectral index can reliably detect deep sedation in mechanically ventilated patients: a prospective multicenter validation study. Anesth Analg 2017; 125: 176-83.

16. Glass PS, Gan TJ, Howell S, Ginsberg B. Drug interactions: volatile anesthetics and opioids. J Clin Anesth 1997; 9(6 Suppl):18S-22S,

17. Mulier JP. Cardiodynamic effects of propofol in comparison to thiopental. Use of the end-systolic pressure-volume relationship and arterial elastance. Anesth Analg 1993; 76: 677-8.

18. Bang JY, Kim S, Choi BM, Kim TY. Pharmacodynamic analysis of the influence of propofol on left ventricular long-axis systolic performance in cardiac surgical patients. J Korean Med Sci 2019; 34: e132.

19. Iwakiri H, Nishihara N, Nagata O, Matsukawa T, Ozaki M, Sessler DI. Individual effect-site concentrations of propofol are similar at loss of consciousness and at awakening. Anesth Analg 2005; 100: 107-10.

20. Bailey JM. Context-sensitive half-times and other decrement times of inhaled anesthetics. Anesth Analg 1997; 85: 681-6.

21. Thomson IR, Moon M, Hudson RJ, Rosenbloom M. Does sufentanil concentration influence isoflurane requirements during coronary artery bypass grafting? J Cardiothorac Vasc Anesth 1999; 13: 9-14.

22. London MJ, Shroyer AL, Coll JR, MaWhinney S, Fullerton DA, Hammermeister KE, et al. Early extubation following cardiac surgery in a veterans population. Anesthesiology 1998; 88: 1447-58.

23. Bhavsar R, Ryhammer PK, Greisen J, Rasmussen LA, Jakobsen CJ. Remifentanil compared with sufentanil does not enhance fast-track possibilities in cardiac surgery-a randomized study. J 
Cardiothorac Vasc Anesth 2016; 30: 1212-20.

24. Svircevic V, Nierich AP, Moons KG, Brandon Bravo Bruinsma GJ, Kalkman CJ, van Dijk D. Fast-track anesthesia and cardiac surgery: a retrospective cohort study of 7989 patients. Anesth Analg 2009; 108: 727-33.

25. Richey M, Mann A, He J, Daon E, Wirtz K, Dalton A, et al. Implementation of an early extubation protocol in cardiac surgical patients decreased ventilator time but not intensive care unit or hospital length of stay. J Cardiothorac Vasc Anesth
2018; 32: 739-44.

26. Dahaba AA. Different conditions that could result in the bispectral index indicating an incorrect hypnotic state. Anesth Analg 2005; 101: 765-73.

27. Lysakowski C, Dumont L, Pellegrini M, Clergue F, Tassonyi E. Effects of fentanyl, alfentanil, remifentanil and sufentanil on loss of consciousness and bispectral index during propofol induction of anaesthesia. Br J Anaesth 2001; 86: 523-7. 